Cinémas

Revue d'études cinématographiques

Journal of Film Studies

\title{
Pragmatique et esthétique du film de mémoire. L'exemple de Strass Café
}

\section{Diane Turcotte}

Volume 6, numéro 2-3, printemps 1996

URI : https://id.erudit.org/iderudit/1000980ar

DOI : https://doi.org/10.7202/1000980ar

Aller au sommaire du numéro

Éditeur(s)

Cinémas

ISSN

1181-6945 (imprimé)

1705-6500 (numérique)

Découvrir la revue

Citer cet article

Turcotte, D. (1996). Pragmatique et esthétique du film de mémoire. L'exemple de Strass Café. Cinémas, 6(2-3), 199-214. https://doi.org/10.7202/1000980ar

\section{Résumé de l'article}

À partir de l'exemple de Strass Café (Léa Pool, Québec, 1980), cet article étudie l'organisation structurelle d'un film de mémoire eu égard à l'instance de réception. Cette approche insiste sut le rôle joué par le spectateur dans la compréhension et l'interprétation du film et les manières dont le texte prévoit une telle participation. Deux aspects sont ainsi privilégiés : la dimension esthétique, par l'analyse des figures mémorielles, et la dimension pragmatique, par l'étude des différents modes de collaboration du spectateur. L'analyse de Strass Café nous permet de mettre de l'avant le concept de « dérive spectatorielle ", qui explique la façon singulière dont le film de mémoire interpelle l'actant-lecteur. 


\title{
Pragmatique et esthétique du film de mémoire. L'exemple de Strass Café
}

\section{Diane Turcotte}

\begin{abstract}
RÉSUMÉ
À partir de l'exemple de Strass Café (Léa Pool, Québec, 1980), cet article étudie l'organisation structurelle d'un film de mémoire eu égard à l'instance de réception. Cette approche insiste sur le rôle joué par le spectateur dans la compréhension et l'interprétation du film et les manières dont le texte prévoit une telle participation. Deux aspects sont ainsi privilégiés: la dimension esthétique, par l'analyse des figures mémorielles, et la dimension pragmatique, par l'étude des différents modes de collaboration du spectateur. L'analyse de Strass Café nous permet de mettre de l'avant le concept de "dérive spectatorielle", qui explique la façon singulière dont le film de mémoire interpelle l'actant-lecteur.
\end{abstract}

\footnotetext{
ABSTRACT

Taking as an example Strass Café (Léa Pool, Quebec, 1980), this article studies the structural organization of a film of memory in relation to its reception. This approach foregrounds the role of the spectator in the understanding and interpretation of the film, and the ways the text anticipates such participation. Two aspects are thus highlighted: the esthetic dimension, through the analysis of memory figures, and the pragmatic dimension, through the study of the different modes of collaboration by the spectator. The analysis of Strass Café enables us to advance the concept of "spectatorial drift" which explains the singular manner in which the film of memory interpellates the actant-reader.
} 
La mémoire au cinéma suggère, en premier lieu, l'image du flash-back, figure emblématique qui a été abondamment utilisée pour illustrer le passé des personnages. D’autres procédés sont venus se greffer au fil des ans pour exprimer le passé, notamment la voix off, l'utilisation du noir et blanc, les images photographiques, etc. Toutefois, à partir de la fin des années 50 , le rendu de la mémoire au cinéma n'exprime plus simplement le passé d'un personnage, mais questionne plutôt la structure filmique et intègre les mécanismes de la mémoire dans sa configuration. Ces films amorcent une réflexion sur le temps, sur les répercussions du passé dans les actions présentes et sur les différentes façons de représenter la mémoire au cinéma. Notre analyse s'attarde justement à un film, Strass Café (Léa Pool, 1980), qui donne à l'acte mémoriel la "conduite du récit " (Deleuze, p. 71), c'est-à-dire où l'organisation structurelle et la dimension esthétique participent à la mise en scène de la mémoire.

Étudier le film de mémoire dans une perspective pragmatique implique, d'une part, l'analyse du dispositif narratif du texte filmique et, d'autre part, l'étude de la problématique de la réception des images filmiques. Ici, nous convoquerons particulièrement la théorie sémio-pragmatique de Roger Odin, qui aborde les déterminismes externes et internes du texte filmique, et la sémiotique de l'interprétation d'Umberto Eco, qui positionne la lecture comme activité de collaboration du spectateur. Ces différentes théories ont en commun l'échange dialectique entre le film et son spectateur, et chacune d'elles privilégie un aspect de cet échange et développe certains concepts qui nous permettront d'étudier comment s'effectue la lecture du film de mémoire. Il s'agit, en fait, d'étudier le rôle joué par le spectateur dans la compréhension et l'interprétation du film de mémoire, et d'analyser les manières dont le texte filmique prévoit une telle participation.

À partir de l'exemple précis de Strass Café, nous analyserons le travail d'actualisation du spectateur. Il faut bien comprendre que tout acte de lecture est, comme le souligne Roger Odin, " [...] une opération mettant en œuvre un système interactif à trois actants: un film $[\ldots]$; une institution $[\ldots]$; et un lecteur, 
[...]» (Odin, 1984, p. 275). Par conséquent, nous examinerons, dans un premier temps, la relation contractuelle entre le texte filmique et son spectateur. Dans un deuxième temps, nous rendrons compte de l'organisation structurelle du film de mémoire qui programme, d'une certaine façon, la compréhension de l'instance spectatorielle. En troisième lieu, nous interrogerons le travail du spectateur qui effectue des propositions de sens car, même si le texte filmique prévoit un certain parcours, il laisse au sujet-spectateur un travail important de coopération. Finalement, nous développerons un nouveau concept, propre à la lecture du film de mémoire, que nous appellerons "dérive spectatorielle".

Avant d'entreprendre l'analyse de Strass Café, il est important de mentionner brièvement ce que nous entendons par film de mémoire. Tout d'abord, le dispositif narratif du film de mémoire comporte quelques critères particuliers qui en font un "genre" difficilement saisissable. Ni tout à fait classique, dans le sens d'un récit linéaire facilement racontable, ni tout à fait expérimental puisqu'il ne s'attarde pas essentiellement à des préoccupations formelles, il se situe davantage du côté de l'hybridité et de la parole. L'évanescence de la mémoire se traduit par l'émiettement narratif et un parti pris pour une manifestation discursive imposante. Alors que le film de fiction classique favorise un effacement de l'énonciation, le film de mémoire l'inscrit plutôt clairement.

La répétition est un autre aspect important du film de mémoire. Certaines expressions ou certaines images sont reprises inlassablement comme pour en extraire les souvenirs et les préciser peu à peu. De plus, la temporalité est floue, discontinue et multiple, plusieurs strates temporelles se côtoient, et ce souvent sans ponctuation à l'intérieur d'un même fragment filmique. Ce type de cinéma rejoint ce que Gilles Deleuze nomme le "cinéma cristallin" où le temps se donne pour lui-même, refusant la transparence de la narration classique par la rupture des liens sensori-moteurs. Ici, la narration ne se limite pas aux actions et aux réactions, elle crée une autre réalité. Gilles Deleuze oppose ce cinéma à un autre régime de l'image, particulièrement présent avant les années 50, qu'il appelle "organique» et dont la 
temporalité est tributaire du mouvement de l'action et des réactions des personnages.

Le film de mémoire est un cinéma de traces plus que d'empreintes (nous ne faisons pas référence à l'empreinte chimique propre à l'image photographique), dans la mesure où la trace est impalpable, évanescente et n'est que le reflet de ce qui subsiste du passé, tandis que l'empreinte renvoie davantage à la durabilité des choses, à des marques tangibles et profondes. Ce sont donc bien des traces que nous retrouvons çà et là à l'intérieur des images du film de mémoire, qui nécessitent de la part du spectateur "l'élaboration mentale de la diégèse " ". Ainsi, de par sa constitution, le film de mémoire demande à être actualisé et interprété par le lecteur, car :

[...] le texte est une machine paresseuse qui exige du lecteur un travail coopératif acharné pour remplir les espaces de non-dit ou de déjà-dit restés en blanc, alors le texte n'est pas autre chose qu'une machine présuppositionnelle (Eco, p. 27).

\section{Strass Café}

Strass Café nous entraîne dans une aventure qui se situe quelque part entre le cadre et le hors-cadre. Les limites de la diégèse nous permettent difficilement d'avoir accès à l'histoire des personnages et fonctionne sous un régime métaphorique, c'està-dire que la diégèse suggère écraniquement, par des images désertes, la mémoire sans souvenir concret. La diégèse au lieu de s'épaissir au fil de l'histoire a tendance à se diluer et même à se raréfier. Des gestes, des regards, des silences si lourds de sens, des lieux de passage qui nous révèlent tout de suite le ton du film. Strass Café pose d'emblée les thèmes de l'errance et de l'incommunicabilité ${ }^{2}$. Toutefois, c'est la problématique de la remémoration qui nous préoccupe à travers l'épaisseur du temps et des souvenirs anesthésiés puisque aucune image ne vient entériner le passé à la fois prégnant et absent.

Le film nous convie à une légende ou à un voyage intérieur : "On dit que c'est une légende. La légende du Strass Café, peutêtre. " La voix poursuit sa litanie et nous assistons à l'errance 
d'une femme - on ne lui donne pas de nom — qui chante au Strass Café et qui quitte un homme pour une femme qui, semble-t-il, lui ressemble. C'est à l'intérieur de cette errance que des bribes de souvenirs font surface, souvenirs que l'on distingue à peine et qui demandent à être déchiffrés. Le souvenir semble resté dans l'ordre du virtuel et ne passe jamais à l'état actuel. Il n'y a pas, dans Strass Café, de représentation du souvenir comme les images du passé qui sont présentées, sous forme de flashback, par exemple. C'est à travers le dire, qui se situe du côté de l'absence et de l'impossible, que se joue le récit.

\section{La relation contractuelle entre le film et son spectateur}

Film d'évocation plutôt que d'action, rythme méditatif, caméra presque autonome, commentaire incantatoire et musique lancinante, Strass Café est une fiction distanciée au même titre que certains films de Chantal Akerman et de Marguerite Duras. D'ailleurs, le film de Léa Pool s'inspire, à plusieurs égards, de l'esthétique développée par l'auteure d'India Song (1974). À l'instar des films de Duras, le film de Léa Pool prend le parti esthétique d'un usage systématique de la voix off, ou plutôt de plusieurs voix qui commentent les images et que l'on ne peut différencier les unes des autres. Ces voix impersonnelles expriment les différentes émotions que ressentent les personnages et en même temps, l'aspect anonyme de ces voix les situe du côté de l'absence et de la passivité. Cette utilisation de la voix off crée une distance entre le film et le spectateur et s'oppose au système standard puisque, comme le souligne Michel Marie, «[...] la parole dominante, dans le cinéma narratif classique, c'est la parole dont la source sonore est représentée dans le champ de l'image" (Marie, p. 41).

Nous pouvons dire que dans Strass Café, le commentaire et la musique viennent combler les manques du récit. La bande visuelle fait place à un dénuement figuratif et au lieu d'enrichir le récit, elle a tendance à le diluer. En fait, c'est la bande-son, et plus particulièrement la chanson qui, sur une musique lancinante de tango, vient raconter et conclure cette histoire désordonnée.

Ainsi, la voix off, qui de par son statut extérieur a tendance, d'une façon standard, à briser l'effet de réel et de présent, 
participe ici plutôt à la construction de la diégèse. En ce sens, c'est le commentaire qui favorise l'opération de diégétisation et qui vient combler les manques du récit comme s'il avait une compréhension des images antérieure à leur présentation. De plus, la musique, même si elle est extra-diégétique, sert aussi l'opération de diégétisation puisqu'elle accompagne la voix off et s'immisce presque à l'intérieur des corps des personnages. Le film utilise le grand potentiel de représentation de la musique, qui apparaît alors quasi diégétique: "Logiquement, elle reste extra-diégétique, mais dans la réalité affective et effective de la réception et du souvenir, elle prend quelque chose de quasi diégétique" (Metz, p.148).

D'autre part, le film de Léa Pool procède, en ce qui a trait à la narrativisation, de deux mouvements contraires. Par sa construction en plans autonomes - hormis les plans de maquillagedémaquillage —, Strass Café refuse, d'une manière générale, la narrativité : "Le traitement en plans autonomes a comme conséquence le blocage de l'établissement d'un rapport de consécution-conséquence entre les plans, c'est-à-dire la négation de la narrativité " (Odin, 1981, p. 151).

Par contre, dès le début du film, nous sommes invités à construire une histoire: "On dit que c'est une légende. La légende du Strass Café, peut-être." À travers les images qui nous sont présentées, nous arrivons à situer les personnages et les actions. Des lieux vides, un port, une rue, Elle vit dans un appartement, Lui marche dans la rue, ils chantent au Strass Café et puis un jour, Elle part vers un autre lieu et lui continue de chanter au Strass Café. Par contre, la bande-image n'est pas suffisante pour construire cette histoire et nous avons besoin de la bande-son pour la compléter. Nous sommes en présence du désir de raconter et simultanément de l'impossibilité de montrer.

L'effet-distance, créé habituellement par l'utilisation systématique de la voix off, est amoindri et presque annihilé par le rôle d'adjuvant de la diégèse que joue justement cette voix off. Toutefois, malgré le fait que le commentaire vienne compléter le minimalisme de la représentation diégétique, Strass Café, de par sa structuration, appelle l'actant-lecteur à collaborer pour établir le lien qui ne figure pas à l'image. Ainsi, l'absence de représenta- 
tion filmique de souvenirs donne une plus grande latitude à l'instance spectatorielle qui répond de ses propres souvenirs à cette absence.

\section{Le système stylistique}

Ici, c'est le commentaire qui encadre le récit. La voix off ne renvoie à aucun personnage de la diégèse mais à une narratrice ou plutôt à plusieurs narratrices non identifiables et qui se trouvent vis-à-vis des images filmiques. Cependant, cette voix provenant d'un autre lieu permet l'enchaînement des plans, puisque le film est construit à partir de plans autonomes. C'est donc la voix off qui régit le récit, qui narre toujours à la troisième personne l'histoire d'Elle et de Lui. "On dit que c'est une légende, la légende du Strass Café, peut-être. Ils sont venus de si loin, ils sont venus de si loin. Elle ne sort presque jamais, elle n'a pas de nom", etc. L'utilisation systématique de la troisième personne rejette la subjectivité et permet une distanciation, car cette voix n'adopte jamais le point de vue d'un personnage de la diégèse. Cette voix qui vient de l'extérieur est le moteur de la narration - elle s'oppose, en quelque sorte, au cinéma de fiction classique pour qui l'essentiel de la parole vient des dialogues et fait partie de la structuration du récit, qui permet un embrayage sur la mémoire: les voix off donnent bien au film " [...] la réverbération du passé comme si la distance du son par rapport à l'image creusait une autre distance, plus fondamentale, avec le présent de la projection. Ce qui se passe devant nous a déjà eu lieu" (Marie, p. 49).

Par ailleurs, nous retrouvons dans Strass Café une disjonction entre les éléments verbaux et le flux visuel. En fait, les images viennent illustrer après coup les propos de la voix off et deviennent une sorte de prolongement de ce qui a été mentionné. Quelquefois, les images précèdent la narration: ce n'est que longtemps après avoir vu en gros plan le réveille-matin qui indique $3 \mathrm{~h} 10$ (plan 15) que nous saurons que toutes les nuits, le train de $3 \mathrm{~h} 10$ la réveille. Il y a comme une répétition, soit de l'image par les mots ou inversement, mais en même temps l'un et l'autre ne viennent jamais lever les ambiguïtés et nous donner plus de détails sur le récit de cette femme. D’ailleurs, le film 
repose sur une sorte d'absence-présence du récit: " [...] tandis que la voix du film s'affirme comme "présence", la singulière articulation du "visuel" et du "narratif" situe clairement le film du côté de l'“absence”" (Longfellow, p. 112).

Strass Café utilise d'autres formes de répétition, que ce soit la répétition à l'intérieur même de l'image tout comme la répétition d'un plan. Il y a aussi la reprise du commentaire à l'intérieur du même plan et d'un plan à l'autre. La récurrence des mots est aussi très présente. Parfois, à l'intérieur d'un même plan, une voix redit ce qu'une autre vient de mentionner, un peu comme en écho. D'un plan à l'autre, c'est le même discours qui se répète en tout ou en partie. La cadence de la voix off nous amène dans une sorte de mémoire, ou plutôt, de souvenirs à moitié oubliés et dont il reste quelques fragments obscurs. Ici, le passé n'est pas illustré par des images, mais il est suggéré par la réitération et la voix off.

Il y a également, dans Strass Café, un jeu incessant sur la temporalité. La narratrice alterne entre le présent, l'imparfait et le conditionnel, chaque mode représentant un aspect de l'héroïne ${ }^{3}$. Le présent est intemporel et sert de description : «Elle est étrangère, elle regarde les mots, les lumières du dehors. " L'imparfait définit une action passée et répétée: "Toutes les nuits, elle allumait les lampes comme si à elle seule, elle décidait du temps, du jour, de la nuit." Enfin, le conditionnel marque le désir: "Elle rêve d'un train qui viendrait la chercher et l'amènerait très loin."

Ce jeu continuel sur la temporalité crée, de façon globale, un brouillage temporel qui fait référence à une errance interminable, une sorte de souvenir évanescent, de secret enfoui dans la mémoire, que l'on pourrait qualifier d'inconscient filmique. Pierre Bayard convoque le concept de hors-temps ${ }^{4}$ pour expliquer ces événements antérieurs temporairement ou définitivement absents, mais qui agissent et que l'on ne peut en quelque sorte atteindre dans le temps filmique. Il s'agit, ici, plus particulièrement de hors-temps absolu, car les "représentations filmiques", qui semblent motiver la progression de la diégèse, ne se retrouveront jamais à l'intérieur du temps filmique. La voix off aussi semble provenir d'un ailleurs insaisissable et fait en sorte 
que " [...] le film n'a plus de prise dans le monde de l'ici et maintenant et suppose en filigrane une inscription dans un hors-lieu et dans un hors-temps" (Pérusse, p. 186).

Au niveau de la composition de l'image, il y a les rails, les champs, les rues illuminées avec les réverbères qui semblent ne plus finir. Ces images qui accusent une profondeur de champ marquée nous invitent à les explorer comme une région du passé, une mémoire lointaine. C'est Gilles Deleuze qui a analysé la profondeur de champ comme fonction de mémorisation et figure de temporalisation. Ces images, tout en ayant l'aspect imperfectif - c'est-à-dire dont le.procès est actuel — nous ramènent à un passé, "une invitation à se souvenir" (Deleuze, p. 143) et dans ce sens mélangent à la fois le présent et le passé. Notre regard est attiré vers le point de fuite, vers le passé en quelque sorte. Tous les plans où Elle se trouve dans son appartement sont composés de telle manière que, par toute la série de fenêtres qui s'ouvrent sur la ville, nous avons une profondeur de champ. De même pour les miroirs placés dans l'appartement, qui en même temps réaffirment l'absence-présence de la mémoire, cette fois-ci par le rapport proche-lointain qu'ils créent. Loin d'être un espace clos, l'appartement, de par ces différentes ouvertures, a aussi cette fonction de vision mémorielle, car « [...] la profondeur de champ crée un certain type d'image-temps directe, qu'on peut définir par la mémoire" (Deleuze, p. 143). Ce n'est pas une mémoire qui se réalise dans les images-souvenir, mais une mémoire qui renferme le temps qui passe avant la constitution des souvenirs.

La mémoire hante le film et chaque intervention musicale vient ponctuer la voix off pour reprendre aussitôt, sorte de répétition interminable, et nous ramener toujours à la préexistence d'un passé peut-être illusoire mais renforcée par sa force d'incantation. Ce son off absolu, qui fait partie du registre poétique, situe le film du côté de la réflexion et non de l'action, comme si l'histoire qui nous est présentée avait déjà eu lieu dans un autre lieu, un autre temps.

\section{Le sujet-spectateur}

Au regard de l'analyse esthétique et des règles institutionnelles, le sujet-spectateur peut amorcer son travail de coopération 
interprétative, car il connaît les parties du texte filmique qu'il doit actualiser et celles qu'il doit mettre entre parenthèses.

L'hypercodage rhétorique et stylistique est ici très significatif au niveau de l'actualisation du film. Le registre poétique est privilégié, par le rythme incantatoire, répétitif et désincarné de la voix off, et nous conduit dans un monde flou où il est difficile de cerner les actions des personnages. Cette forme de narration "[...] constitue le principe structurel même de l'articulation du film" (Longfellow, p. 106). De plus, la temporalité est imprécise et indéterminée - polyvalence des temps de verbe - et fait référence à une temporalité mémorielle et circulaire. Ce parti pris esthétique crée une distance entre le texte filmique et l'actant-lecteur.

Afin d'élaborer son système stylistique, l'Auteur Modèle, pour employer la terminologie d'Umberto Eco, se réfere à un type d'encyclopédie auquel le Lecteur Modèle doit aussi se rapporter. Examinons, tout d'abord, les inférences de scénarios communs suggérés par le contexte. Strass Café fait appel à une mobilité géographique et spatiale en privilégiant des lieux de passage: un port, un fleuve, un train, un champ désert, une gare. Le discours verbal aussi s'attarde particulièrement à ces lieux singuliers: "Elle parlait toujours d'une gare avec des portes dans toutes les directions pour aller n'importe où, d'une gare désaffectée où l'herbe a mangé les rails, plus aucun train ne passe" (plan 16) et "Elle regarde les eaux du fleuve, ne s'arrête pas, dans aucun port, jamais" (plans 23-24). Cette mobilité spatiale, évoquée par des lieux de passage, est mise en opposition par l'utilisation de plans fixes très longs. Dans l'image, nous retrouvons une dualité entre mobilité-immobilité, qui se compare à celle du discours verbal entre vouloir oublier et se remémorer: "Elle parcourt l'oubli dans sa tête, à la recherche du vide, de la mémoire du vide. Elle est pleine de murs" (plan 34) et "Le train de $3 \mathrm{~h} 10$, tout le monde s'en souvient, on voudrait tout oublier, ne jamais avoir su, elle aurait aimé savoir " (plan 21). Cette dualité entre mobilité-immobilité est ici présentée en référence à l'exil et à cette volonté d'oublier et de se souvenir.

L'expérience d'autres textes - pour ceux qui les connaissent, bien entendu - comme ceux de Marguerite Duras et particu- 
lièrement India Song nous avait habituée à l'utilisation d'une voix off systématique ainsi qu'à cet effet de répétition et de raréfaction de la diégèse. De plus, nous pouvons effectuer un rapprochement avec la littérature féministe qui a développé un style singulier axé sur la parole:

[...] dans cette prise de parole lyrique, répétitive, poétique, faisant fi du discours rationnel. Cette voix off féminine, souveraine, couplée à une absence de figuration ou de corps, semble également être une des clés de voûte d'une certaine pratique cinématographique féministe et l'antidote le plus radical aux pouvoirs de fascination de l'image (Pérusse, p. 191).

De même, ce commentaire off qui renferme plusieurs voix enlève, selon Pascal Bonitzer, ce côté autoritaire que l'on accorde à un seul narrateur et que l'on retrouve dans le cinéma standard. Également, le fait d'utiliser des voix féminines comme narratrices, rôle dévolu presque essentiellement aux hommes, s'inscrit très bien à l'intérieur d'une pratique cinématographique féministe.

À la lumière de ces inférences, il est plus facile pour l'actantlecteur d'effectuer une lecture de Strass Café se rapprochant le plus de celle générée par l'Auteur Modèle. Par contre, le texte filmique étant particulièrement fuyant, la détermination de la fabula, c'est-à-dire du schéma de la narration, n'est pas facilement saisissable. Toutefois, nous croyons que la logique du texte est maintenue par cette mémoire latente, ces souvenirs absents, ce passé qui plane littéralement sur tout le déroulement du film et qui, tout en étant absent, motive la progression, si l'on peut parler de progression, de l'histoire ou plutôt du temps qui passe malgré tout. En fait, le texte filmique recèle une "passéité" dans une contemporanéité.

Le monde possible, construit par la structure narrative, est celui de l'errance d'une femme "à la recherche d'un lieu qu'elle pourrait habiter", mais qui est inexorablement repoussé par les souvenirs d'un autre temps qui la hantent, sorte de mémoire involontaire qui ne prend jamais forme dans la structure filmique et qui est, cependant, constamment évoquée à travers le système stylistique. Toujours ce désir de montrer l'«irreprésentable ". 
La structuration du récit de Strass Café joue sur cette absence de représentation filmique et en fait l'énoncé principal du film.

Avec cette structure ouverte et l'absence de représentation filmique du passé, Strass Café laisse une place importante à l'instance spectatorielle au niveau de sa lecture, puisque son activité d'interprétation se situe presque au-delà du texte filmique. Étant davantage du côté de l'évanescence que de la matérialité, l'organisation du récit permet à chaque actant-lecteur d'y trouver son espace et d'y faire intervenir sa propre mémoire.

À partir de l'analyse de Strass Café, nous constatons que c'est l'expérience du temps et de la mémoire qui est l'enjeu même de l'organisation structurelle et par conséquent la "conduite du récit». Tout repose sur le statut de la trace, c'est-à-dire d'une mémoire à déchiffrer où le locuteur est porté à relancer certains fragments de l'histoire reçue pour recevoir un autre fragment en écho. La mémoire ainsi éveillée favorise cette dynamique entre le film et l'actant-lecteur à l'image de la dialectique entre la mémoire et l'oubli.

Ainsi, le film de mémoire n'apporte pas seulement une réflexion sur le contenu des énoncés et les figures permettant un embrayage sur les souvenirs, mais suscite également une réflexion entre énoncé et énonciation. Chacune de ces instances vient appuyer l'autre et perd ainsi son autonomie au profit de l'ensemble mémoriel. En fait, si la réflexion demeurait au niveau de l'énoncé, nous serions davantage du côté de la simple incursion dans le passé des personnages que dans la véritable conduite du récit. Tandis qu'ici, l'énonciation se trouve à être l'enveloppe mémorielle, en quelque sorte, et elle questionne, autant que l'énoncé, le fonctionnement de la mémoire par son architecture globale. Effectivement, les diverses figures stylistiques " [...] mettent en lumière les mécanismes constitutifs d'un film, ses conditions d'existence: sans abandonner le plan de l'histoire, ils montrent le travail du discours" (Casetti, p. 181). Ici, l'énonciation filmique se trouve à commenter, par son travail discursif, l'énoncé.

La forme narrative du film de mémoire fonctionne ainsi à l'image de la mémoire en acte. L'aspect fragmentaire, répétitif, elliptique et lacunaire s'inscrit clairement au détriment de la 
cohérence logique de l'histoire. Ce parti pris discursif favorise la reconstruction et la représentation et met au premier plan le processus du film et simultanément le processus de la mémoire. C'est d'ailleurs ce travail sur le dispositif narratif qui nous amène à évoquer l'hypothèse de la "dérive spectatorielle".

La relation qui s'installe entre le film de mémoire et l'actantlecteur est tout à fait particulière, puisqu'elle favorise à la fois le fonctionnement des opérations de fictionnalisation, c'est-à-dire la croyance en ce monde fictionnel, et la création d'une distance qui renvoie le spectateur à sa propre expérience mémorielle. Cette distance et cette fascination nous amènent à aborder ce que nous appelons la "dérive spectatorielle», particularité qui distingue les films de mémoire des films de fiction classique. Pour nous la "dérive spectatorielle" est cette opération qui fait que le sujet-spectateur, tout en étant fasciné par ce qui se passe à l'écran, s'abandonne à sa propre mémoire. En fait, il est interpellé par la structure filmique qui représente l'acte mémoriel. Le spectateur répond par les événements de son expérience à la construction filmique effectuée par l'auteur. Par conséquent, la dynamique entre le film et l'actant-lecteur s'apparente à une structure en écho puisque nous avons affaire à une répétition différée de l'acte mémoriel dans le surgissement des souvenirs de l'actant-lecteur.

La construction du film montre l'aptitude à se souvenir et à chercher, hors du texte, l'essence même du film. Représenter la mémoire ne consiste pas seulement à inclure des images du passé des personnages concernés, mais à rendre présents les mécanismes de la mémoire ; c’est suggérer ce qui était avant et montrer le processus nécessaire à la création des imagessouvenir. Pour Gilles Deleuze:

Il appartient au cinéma de saisir ce passé et ce futur qui coexistent avec l'image présente. Filmer ce qui est avant et ce qui est après... Peut-être faut-il faire passer à l'intérieur du film ce qui est avant le film, et après le film, pour sortir de la chaîne des présents (Deleuze, p. 55).

Strass Café arrive justement à rendre tangible la mémoire qui correspond à ce que l'on pourrait appeler l'"avant diégétique». 
C'est à travers l'organisation structurelle, la profondeur de champ, la voix off, la charge émotive des personnages, que ce film accède à une représentation directe de la mémoire et à la création d'un hors-temps.

Ainsi, le concept de hors-temps qui correspond aux représentations filmiques absentes, ayant une portée diégétique importante, favorise la poussée hors du texte et appuie la notion de "dérive spectatorielle". Cette dernière, tout en étant extérieure au texte filmique, est une conséquence de la lecture de ce type de film, c'est-à-dire qu'elle se construit sous l'impulsion du texte.

La structuration du film de mémoire, par ses nombreuses lacunes, ses multiples intervalles, son aspect fragmentaire, favorise un jeu d'échos avec son spectateur. La relation pragmatique qui existe entre le film de mémoire et son sujet-spectateur est plus évidente qu'avec les films de fiction classique, car l'actantlecteur est plus sollicité dans la compréhension du texte filmique. Ici, l'histoire ne se raconte pas d'elle-même, elle a besoin que quelqu'un l'aide à fonctionner. De plus, le texte filmique est construit de telle sorte que par moments, il s'efface au profit d'un au-delà, cette fois-ci, plus personnel: "Le spectateur, en somme, s'engage à regarder: il répond à la disponibilité du monde écranique par une véritable vocation; il répond à la proposition d'une destination, en assumant ses propres responsabilités" (Casetti, p. 25).

Le Lecteur Modèle suit le trajet de la trace à déchiffrer et c'est à partir de la construction du texte filmique qu'il effectue son travail de coopération interprétative. Il a, dans un premier temps, à actualiser le texte filmique à travers les propriétés structurales et le système stylistique, ce qui l'amène à un premier niveau de pertinence (l'interprétation du récit) et, dans un deuxième temps, ces mêmes propriétés incitent l'actant-lecteur à un deuxième niveau de signifiance, si on peut dire, qui fait surgir sa mémoire, elle aussi enfouie dans les lieux de l'oubli. Le second trajet répète, en quelque sorte, le parcours filmique initial.

Le film de mémoire construit à partir de ce double mouvement, intérieur et extérieur, interpelle donc différemment le sujet-spectateur du cinéma de fiction standard. Par conséquent, 
l'accès du spectateur au texte filmique est-il double: le savoir fictionnalisant, puisque le film de mémoire fait partie du cinéma de fiction, et la "dérive spectatorielle" qui le singularise. En fait, la structure du film de mémoire épouse, à travers la fascination et la distance qu'elle crée, la dualité du discours qu'elle propose, celle de la mémoire et de l'oubli.

Ainsi, la coopération interprétative du film de mémoire demande à l'actant-lecteur une double lecture, car au-delà de la compréhension diégétique, le texte filmique trouve sa véritable complétude dans la mémoire qu'il éveille.

C'est donc sur les traces de l'oubli, du fragment et d'une mémoire à déchiffrer que le sujet-spectateur est amené à produire le sens du film et à se livrer à une "[...] expérience nouvelle du temps et de la mémoire" (Schefer, p. 14).

\section{Collège André-Laurendeau}

\section{NOTES}

1 Nous reprenons ici l'expression de Roger Odin lorsqu'il analyse le film de famille dans le troisième volume de sa thèse de doctorat: L'Analyse sémiologique des films. Vers une sémio-pragmatique (Paris: École des hautes études en sciences sociales, 1982).

2 Ces aspects ont été étudiés par Denise Pérusse à l'intérieur de sa thèse de doctorat La Mise en espace-temps des femmes dans le cinéma québécois de 1976 à 1986 (Sainte-Foy: Université Laval, 1989).

3 Pour une analyse plus détaillée de l'articulation temporelle de Strass Café, voir Denise Pérusse, "Strass Café, une esthétique basée sur le dénuement de la figuration et sur la souveraineté de la voix ", La Mise en espace-temps des femmes dans le cinéma québécois de 1976 à 1986 (Sainte-Foy: Université Laval, 1989, p. 171-172).

4 Cette notion de hors-temps est développée dans l'article "Le hors-temps " paru dans Hors Cadre, n 9 (1991) p. 135-147.

\section{OUVRAGES CITÉS}

Casetti, Francesco. D'un regard l'autre. Le film et son spectateur. Lyon: Presses Universitaires de Lyon, 1990.

Deleuze, Gilles. L'Image-temps. Paris: Minuit, 1985.

Eco, Umberto. Lector in fabula. Paris: Grasset, 1985.

Longfellow, Brenda. "L'écriture féministe de Journal Inachevé et Strass Café», dans Michel Larouche (direction), "Cinéma québécois. Nouveaux courants, nouvelles critiques". Dérives, n 52 (1986) p. 103-116.

Marie, Michel. "La parole dans le cinéma français contemporain: l'exemple d'India Song». Ça Cinéma, n 19 (1980) p. 37-49. 
Metz, Christian. L'Énonciation impersonnelle ou le site du film. Paris: Méridiens Klincksieck, 1991.

Odin, Roger. "Film documentaire. Lecture documentarisante». Cinémas et réalités (collectif). Paris: Université de Ste-Étienne/Travaux XLI/Centre interdisciplinaire sur l'expression contemporaine (1984) p. 263-278.

Odin, Roger. «Le film de fiction menacé par la photographie et sauvé par la bandeson ", dans D. Chateau, A. Gardies, F. Jost (direction), Cinémas de la modernité: films, théories. Paris: Klincksieck (1981) p. 147-171.

Pérusse, Denise. "Strass Café, une esthétique basée sur le dénuement de la figuration et sur la souveraineté de la voix". La Mise en espace-temps des femmes dans le cinéma québécois de 1976 à 1986 (thèse de doctorat). Sainte-Foy: Université Laval (1989) p. 166-194.

Schefer, J. L. L'Homme ordinaire du cinéma. Paris: Cahiers du Cinéma/Gallimard, 1980. 